\title{
Effect of the minimal length uncertainty relation on the density of states and the cosmological constant problem
}

\author{
Lay Nam Chang, ${ }^{*}$ Djordje Minic, ${ }^{\dagger}$ Naotoshi Okamura, ${ }^{\ddagger}$ and Tatsu Takeuchi ${ }^{\S}$ \\ Institute for Particle Physics and Astrophysics, Physics Department, Virginia Tech, Blacksburg, Virginia 24061
}

(Received 9 January 2002; published 19 June 2002)

\begin{abstract}
We investigate the effect of the minimal length uncertainty relation, motivated by perturbative string theory, on the density of states in momentum space. The relation is implemented through the modified commutation relation $\left[\hat{x}_{i}, \hat{p}_{j}\right]=i \hbar\left[\left(1+\beta \hat{p}^{2}\right) \delta_{i j}+\beta^{\prime} \hat{p}_{i} \hat{p}_{j}\right]$. We point out that this relation, which is an example of a UV/IR relation, implies the finiteness of the cosmological constant. While our result does not solve the cosmological constant problem, it does shed new light on the relation between this outstanding problem and UV/IR correspondence. We also point out that the blackbody radiation spectrum will be modified at higher frequencies, but the effect is too small to be observed in the cosmic microwave background spectrum.
\end{abstract}

DOI: 10.1103/PhysRevD.65.125028

PACS number(s): 98.80.Es, 02.40.Gh, 03.65.Sq, 98.70.Vc

\section{INTRODUCTION}

In this paper, we continue our investigation [1] of the consequences of the commutation relation

$$
[\hat{x}, \hat{p}]=i \hbar\left(1+\beta \hat{p}^{2}\right),
$$

which leads to the minimal length uncertainty relation

$$
\Delta x \geqslant \frac{\hbar}{2}\left(\frac{1}{\Delta p}+\beta \Delta p\right) .
$$

As reviewed in Ref. [1], Eq. (2) has appeared in the context of perturbative string theory [2] where it is implicit in the fact that strings cannot probe distances below the string scale $\hbar \sqrt{\beta}$. It should be noted that the precise theoretical framework for such a minimal length uncertainty relation is not understood in string theory. In particular, it is not clear whether Eq. (1) represents the correct quantum mechanical implementation of Eq. (2). Indeed, Kempf has shown that the commutation relation which implies the existence of a minimal length is not unique [3].

Furthermore, Eq. (2) does not seem to be universally valid. For example, both in the realms of perturbative and nonperturbative string theory (where distances shorter than the string scale can be probed by $D$-branes [4]), another type of uncertainty relation involving both spatial and time coordinates has been found to hold [5]. The distinction (and relation) between the minimal length uncertainty relation and the space-time uncertainty relation has been clearly emphasized by Yoneya [6].

Notwithstanding these caveats, the minimum length uncertainty formula does exhibit the basic features of UV/IR correspondence: when $\Delta p$ is large, $\Delta x$ is proportional to $\Delta p$, a fact which seems counterintuitive from the point of view of local quantum field theory. As is well known, this kind of

\footnotetext{
*Electronic address: laynam@vt.edu

†Electronic address: dminic@vt.edu

¥Electronic address: nokamura@vt.edu

${ }^{\S}$ Electronic address: takeuchi@vt.edu
}

UV/IR correspondence has been previously encountered in various contexts: the AdS/CFT correspondence [7], noncommutative field theory [8], and more recently in the attempts to understand quantum gravity in asymptotically de Sitter spaces $[9,10]$.

Moreover, it has been argued by various authors [11] that the UV/IR correspondence, described by Eq. (2), is relevant for the understanding of the cosmological constant problem [12]. Likewise, it has been suggested in the literature that some kind of UV/IR relation is necessary to understand observable implications of short distance physics on inflationary cosmology $[3,13]$.

In this paper we ask the question whether the cosmological constant problem could be understood by utilizing a concrete UV/IR relation, such as Eq. (1). In particular, we study the implication of the commutation relation on the effective density of states in the vacuum and consequently on the cosmological constant problem. We point out that the commutation relation implies the finiteness of the cosmological constant and the modification of the blackbody radiation spectrum. While we do not present a solution to the cosmological constant problem, our results offer a new perspective from which this outstanding problem may be addressed.

\section{THE CLASSICAL LIMIT AND THE LIOUVILLE THEOREM}

The observation we would like to make is that the righthand side of Eq. (1) can be considered to define an "effective" value of $\hbar$ which is $p$ dependent. This means that the size of the unit cell that each quantum state occupies in phase space can be thought of as being also $p$ dependent. This will change the $p$ dependence of the density of states and affect the calculation of the cosmological constant, the blackbody radiation spectrum, etc. [14]. For this interpretation to make sense, we must first check that any volume of phase space evolves in such a way that the number of states inside does not change with time. What we are looking for here is the analog of the Liouville theorem. To place the discussion in a general context, we begin by extending Eq. (1) to higher dimensions. 

[15]

In $D$ dimensions, Eq. (1) is extended to the tensorial form

$$
\left[\hat{x}_{i}, \hat{p}_{j}\right]=i \hbar\left(\delta_{i j}+\beta \hat{p}^{2} \delta_{i j}+\beta^{\prime} \hat{p}_{i} \hat{p}_{j}\right)
$$

If the components of the momentum $\hat{p}_{i}$ are assumed to commute with each other,

$$
\left[\hat{p}_{i}, \hat{p}_{j}\right]=0
$$

then the commutation relations among the coordinates $\hat{x}_{i}$ are almost uniquely determined by the Jacobi identity (up to possible extensions) as

$$
\left[\hat{x}_{i}, \hat{x}_{j}\right]=i \hbar \frac{\left(2 \beta-\beta^{\prime}\right)+\left(2 \beta+\beta^{\prime}\right) \beta \hat{p}^{2}}{\left(1+\beta \hat{p}^{2}\right)}\left(\hat{p}_{i} \hat{x}_{j}-\hat{p}_{j} \hat{x}_{i}\right) .
$$

Let us take a look at what happens in the classical limit. Recall that the quantum mechanical commutator corresponds to the Poisson bracket in classical mechanics via

$$
\frac{1}{i \hbar}[\hat{A}, \hat{B}] \Rightarrow\{A, B\}
$$

So the classical limits of Eqs. (3)-(5) read

$$
\begin{aligned}
& \left\{x_{i}, p_{j}\right\}=\left(1+\beta p^{2}\right) \delta_{i j}+\beta^{\prime} p_{i} p_{j}, \\
& \left\{p_{i}, p_{j}\right\}=0, \\
& \left\{x_{i}, x_{j}\right\}=\frac{\left(2 \beta-\beta^{\prime}\right)+\left(2 \beta+\beta^{\prime}\right) \beta p^{2}}{\left(1+\beta p^{2}\right)}\left(p_{i} x_{j}-p_{j} x_{i}\right) .
\end{aligned}
$$

The time evolutions of the coordinates and momenta are governed by

$$
\begin{aligned}
& \dot{x}_{i}=\left\{x_{i}, H\right\}=\left\{x_{i}, p_{j}\right\} \frac{\partial H}{\partial p_{j}}+\left\{x_{i}, x_{j}\right\} \frac{\partial H}{\partial x_{j}}, \\
& \dot{p}_{i}=\left\{p_{i}, H\right\}=-\left\{x_{j}, p_{i}\right\} \frac{\partial H}{\partial x_{j}} .
\end{aligned}
$$

The analog of the Liouville theorem in this case states that the weighted phase space volume

$$
\frac{d^{D} \mathbf{x} d^{D} \mathbf{p}}{\left[1+\beta p^{2}\right]^{D-1}\left[1+\left(\beta+\beta^{\prime}\right) p^{2}\right]^{1-\beta^{\prime} / 2\left(\beta+\beta^{\prime}\right)}}
$$

is invariant under time evolution. To see this, consider an infinitesimal time interval $\delta t$. The evolution of the coordinates and momenta during $\delta t$ are

$$
\begin{aligned}
& x_{i}^{\prime}=x_{i}+\delta x_{i}, \\
& p_{i}^{\prime}=p_{i}+\delta p_{i},
\end{aligned}
$$

$$
\begin{aligned}
& \delta x_{i}=\left[\left\{x_{i}, p_{j}\right\} \frac{\partial H}{\partial p_{j}}+\left\{x_{i}, x_{j}\right\} \frac{\partial H}{\partial x_{j}}\right] \delta t, \\
& \delta p_{i}=\left[-\left\{x_{j}, p_{i}\right\} \frac{\partial H}{\partial x_{j}}\right] \delta t .
\end{aligned}
$$

An infinitesimal phase space volume after this infinitesimal evolution is

$$
d^{D} \mathbf{x}^{\prime} d^{D} \mathbf{p}^{\prime}=\left|\frac{\partial\left(x_{1}^{\prime}, \ldots, x_{D}^{\prime}, p_{1}^{\prime}, \ldots, p_{D}^{\prime}\right)}{\partial\left(x_{1}, \ldots, x_{D}, p_{1}, \ldots, p_{D}\right)}\right| d^{D} \mathbf{x} d^{D} \mathbf{p} .
$$

Since

$$
\begin{aligned}
& \frac{\partial x_{i}^{\prime}}{\partial x_{j}}=\delta_{i j}+\frac{\partial \delta x_{i}}{\partial x_{j}}, \quad \frac{\partial x_{i}^{\prime}}{\partial p_{j}}=\frac{\partial \delta x_{i}}{\partial p_{j}}, \\
& \frac{\partial p_{i}^{\prime}}{\partial x_{j}}=\frac{\partial \delta p_{i}}{\partial x_{j}}, \quad \frac{\partial p_{i}^{\prime}}{\partial p_{j}}=\delta_{i j}+\frac{\partial \delta p_{i}}{\partial p_{j}}
\end{aligned}
$$

the Jacobian to first order in $\delta t$ is

$$
\left|\frac{\partial\left(x_{1}^{\prime}, \ldots, x_{D}^{\prime}, p_{1}^{\prime}, \ldots, p_{D}^{\prime}\right)}{\partial\left(x_{1}, \ldots, x_{D}, p_{1}, \ldots, p_{D}\right)}\right|=1+\left(\frac{\partial \delta x_{i}}{\partial x_{i}}+\frac{\partial \delta p_{i}}{\partial p_{i}}\right)+\cdots
$$

We find

$$
\begin{aligned}
\left(\frac{\partial \delta x_{i}}{\partial x_{i}}+\frac{\partial \delta p_{i}}{\partial p_{i}}\right) \frac{1}{\delta t} & \frac{\partial}{\partial x_{i}}\left[\left\{x_{i}, p_{j}\right\} \frac{\partial H}{\partial p_{j}}+\left\{x_{i}, x_{j}\right\} \frac{\partial H}{\partial x_{j}}\right]-\frac{\partial}{\partial p_{i}}\left[\left\{x_{j}, p_{i}\right\} \frac{\partial H}{\partial x_{j}}\right] \\
= & {\left[\frac{\partial}{\partial x_{i}}\left\{x_{i}, p_{j}\right\}\right] \frac{\partial H}{\partial p_{j}}+\left\{x_{i}, p_{j}\right\} \frac{\partial^{2} H}{\partial x_{i} \partial p_{j}}+\left[\frac{\partial}{\partial x_{i}}\left\{x_{i}, x_{j}\right\}\right] \frac{\partial H}{\partial x_{j}} } \\
& +\left\{x_{i}, x_{j}\right\} \frac{\partial^{2} H}{\partial x_{i} \partial x_{j}}-\left[\frac{\partial}{\partial p_{i}}\left\{x_{j}, p_{i}\right\}\right] \frac{\partial H}{\partial x_{j}}-\left\{x_{j}, p_{i}\right\} \frac{\partial^{2} H}{\partial p_{j} \partial x_{i}} \\
= & {\left[\frac{\partial}{\partial x_{i}}\left\{x_{i}, x_{j}\right\}\right] \frac{\partial H}{\partial x_{j}}-\left[\frac{\partial}{\partial p_{i}}\left\{x_{j}, p_{i}\right\}\right] \frac{\partial H}{\partial x_{j}} } \\
= & {\left[-\frac{\left(2 \beta-\beta^{\prime}\right)+\left(2 \beta+\beta^{\prime}\right) \beta p^{2}}{\left(1+\beta p^{2}\right)}(D-1) p_{j}\right] \frac{\partial H}{\partial x_{j}} } \\
& -\left[\left(2 \beta+D \beta \beta^{\prime}\right) p_{j} \frac{\partial H}{\partial x_{j}}\right. \\
& -\left[2(D-1) \beta\left(\frac{1+\left(\beta+\beta^{\prime}\right) p^{2}}{1+\beta p^{2}}\right)+\left(2 \beta+\beta^{\prime}\right)\right] p_{j} \frac{\partial H}{\partial x_{j}}
\end{aligned}
$$

Therefore, to first order in $\delta t$

with 


$$
\begin{aligned}
d^{D} \mathbf{x}^{\prime} d^{D} \mathbf{p}^{\prime}= & d^{D} \mathbf{x} d^{D} \mathbf{p}\left[1-\left\{2(D-1) \beta\left(\frac{1+\left(\beta+\beta^{\prime}\right) p^{2}}{1+\beta p^{2}}\right)\right.\right. \\
& \left.\left.+\left(2 \beta+\beta^{\prime}\right)\right\} p_{j} \frac{\partial H}{\partial x_{j}} \delta t\right] .
\end{aligned}
$$

On the other hand,

$$
\begin{aligned}
1+\beta p^{\prime 2}= & 1+\beta\left(p_{i}+\delta p_{i}\right)^{2} \\
= & 1+\beta\left(p^{2}+2 p_{i} \delta p_{i}+\cdots\right) \\
= & 1+\beta\left(p^{2}-2 p_{i}\left\{x_{i}, p_{j}\right\} \frac{\partial H}{\partial x_{j}} \delta t+\cdots\right) \\
= & 1+\beta\left(p^{2}-2\left[1+\left(\beta+\beta^{\prime}\right) p^{2}\right] p_{j} \frac{\partial H}{\partial x_{j}} \delta t+\cdots\right) \\
= & \left(1+\beta p^{2}\right)-2 \beta\left[1+\left(\beta+\beta^{\prime}\right) p^{2}\right] p_{j} \frac{\partial H}{\partial x_{j}} \delta t+\cdots \\
= & \left(1+\beta p^{2}\right)[1 \\
& \left.-2 \beta\left(\frac{1+\left(\beta+\beta^{\prime}\right) p^{2}}{1+\beta p^{2}}\right) p_{j} \frac{\partial H}{\partial x_{j}} \delta t+\cdots\right]
\end{aligned}
$$

and

$$
\begin{aligned}
1+ & \left(\beta+\beta^{\prime}\right) p^{\prime 2} \\
& =1+\left(\beta+\beta^{\prime}\right)\left(p_{i}+\delta p_{i}\right)^{2} \\
& =1+\left(\beta+\beta^{\prime}\right)\left(p^{2}+2 p_{i} \delta p_{i}+\cdots\right) \\
& =1+\left(\beta+\beta^{\prime}\right)\left(p^{2}-2 p_{i}\left\{x_{i}, p_{j}\right\} \frac{\partial H}{\partial x_{j}} \delta t+\cdots\right) \\
& =1+\left(\beta+\beta^{\prime}\right)\left(p^{2}\right. \\
& \left.-2\left[1+\left(\beta+\beta^{\prime}\right) p^{2}\right] p_{j} \frac{\partial H}{\partial x_{j}} \delta t+\cdots\right) \\
& =\left[1+\left(\beta+\beta^{\prime}\right) p^{2}\right] \\
& -2\left(\beta+\beta^{\prime}\right)\left[1+\left(\beta+\beta^{\prime}\right) p^{2}\right] p_{j} \frac{\partial H}{\partial x_{j}} \delta t+\cdots \\
& =\left[1+\left(\beta+\beta^{\prime}\right) p^{2}\right]\left[1-2\left(\beta+\beta^{\prime}\right) p_{j} \frac{\partial H}{\partial x_{j}} \delta t+\cdots\right] .
\end{aligned}
$$

Therefore, to first order in $\delta t$

$$
\begin{aligned}
{\left[1+\beta p^{\prime 2}\right]^{-D+1}\left[1+\left(\beta+\beta^{\prime}\right) p^{\prime 2}\right]^{-1+\beta^{\prime} / 2\left(\beta+\beta^{\prime}\right)} } & \\
= & {\left[1+\beta p^{2}\right]^{-D+1}\left[1+\left(\beta+\beta^{\prime}\right) p^{2}\right]^{-1+\beta^{\prime} / 2\left(\beta+\beta^{\prime}\right)} } \\
& \times\left[1+\left\{2(D-1) \beta\left(\frac{1+\left(\beta+\beta^{\prime}\right) p^{2}}{1+\beta p^{2}}\right)\right.\right. \\
+ & \left.\left.2\left(\beta+\beta^{\prime}\right)\left(1-\frac{\beta^{\prime}}{2\left(\beta+\beta^{\prime}\right)}\right)\right\} p_{j} \frac{\partial H}{\partial x_{j}} \delta t\right] \\
= & {\left[1+\beta p^{2}\right]^{-D+1}\left[1+\left(\beta+\beta^{\prime}\right) p^{2}\right]^{-1+\beta^{\prime} / 2\left(\beta+\beta^{\prime}\right)} } \\
& \times\left[1+\left\{2(D-1) \beta\left(\frac{1+\left(\beta+\beta^{\prime}\right) p^{2}}{1+\beta p^{2}}\right)\right.\right. \\
& \left.\left.+\left(2 \beta+\beta^{\prime}\right)\right\} p_{j} \frac{\partial H}{\partial x_{j}} \delta t\right] .
\end{aligned}
$$

From Eqs. (16) and (19), we deduce that the weighted phase space volume Eq. (9) is invariant. Note that in addition to the noncanonical Poisson brackets between the coordinates and momenta, those among the coordinates themselves (and thus the noncommutative geometry of the problem) are crucial in arriving at this result. When $\beta^{\prime}=0$, Eq. (9) simplifies to

$$
\frac{d^{D} \mathbf{x} d^{D} \mathbf{p}}{\left(1+\beta p^{2}\right)^{D}}
$$

As a concrete example, consider the 1D harmonic oscillator with the Hamiltonian

$$
H=\frac{p^{2}}{2 \mu}+\frac{1}{2} \mu \omega^{2} x^{2}
$$

The equations of motion are

$$
\begin{aligned}
& \dot{x}=\{x, H\}=\frac{1}{\mu}\left(1+\beta p^{2}\right) p, \\
& \dot{p}=\{p, H\}=-\mu \omega^{2}\left(1+\beta p^{2}\right) x .
\end{aligned}
$$

These equations can be solved to yield

$$
\begin{aligned}
& x(t)=x_{\max } \sqrt{1+\varepsilon} \frac{\sin (\sqrt{1+\varepsilon} \omega t)}{\sqrt{1+\varepsilon \sin ^{2}(\sqrt{1+\varepsilon} \omega t)}}, \\
& p(t)=p_{\max } \frac{\cos (\sqrt{1+\varepsilon} \omega t)}{\sqrt{1+\varepsilon \sin ^{2}(\sqrt{1+\varepsilon} \omega t)}},
\end{aligned}
$$

where

$$
\varepsilon=2 \mu E \beta, \quad x_{\max }=\sqrt{\frac{2 E}{\mu \omega^{2}}}, \quad p_{\max }=\sqrt{2 \mu E} .
$$

Note that the period of oscillation, $T$, is now energy (and thus amplitude) dependent: 


$$
T=\frac{2 \pi}{\omega \sqrt{1+\varepsilon}} .
$$

Now, consider the infinitesimal phase space volume sandwiched between the equal-energy contours $E$ and $E+d E$, and the equal-time contours $t$ and $t+d t$. It is straightforward to show that

$$
d E d t=\frac{d x d p}{1+\beta p^{2}} .
$$

The left-hand side of this equation is time-independent by definition, so the right-hand side must be also.

Finally, note that the semi classical quantization $(\hbar \rightarrow 0$ limit) of the harmonic oscillator is consistent with the full quantum mechanical result derived in our previous paper, Ref. [1].

\section{DENSITY OF STATES}

From this point on, we will only consider the $\beta^{\prime}=0$ case for the sake of simplicity.

Integrating over the coordinates, the invariant phase space volume Eq. (20) becomes

$$
\frac{V d^{D} \mathbf{p}}{\left(1+\beta p^{2}\right)^{D}}
$$

where $V$ is the coordinate space volume. This implies that upon quantization, the number of quantum states per momentum space volume should be assumed to be

$$
\frac{V}{(2 \pi \hbar)^{D}} \frac{d^{D} \mathbf{p}}{\left(1+\beta p^{2}\right)^{D}} .
$$

Equation (28) indicates that the density of states in momentum space must be modified by the extra factor of (1 $\left.+\beta p^{2}\right)^{-D}$. This factor effectively cuts off the integral beyond $p=1 / \sqrt{\beta}$. Indeed, in $3 \mathrm{D}$ the weight factor is

$$
\frac{1}{\left(1+\beta p^{2}\right)^{3}},
$$

the plot of which is shown in Fig. 1. We look at the consequence of this modification in the calculation of the cosmological constant and the blackbody radiation spectrum in the following.

\section{A. The cosmological constant}

The cosmological constant is obtained by summing over the zero-point fluctuation energies of harmonic oscillators, each of which corresponds to a particular particle momentum state [12]. If we assume that the zero-point energy of each oscillator is of the usual, canonical, form

$$
\frac{1}{2} \hbar \omega=\frac{1}{2} \sqrt{p^{2}+m^{2}}
$$

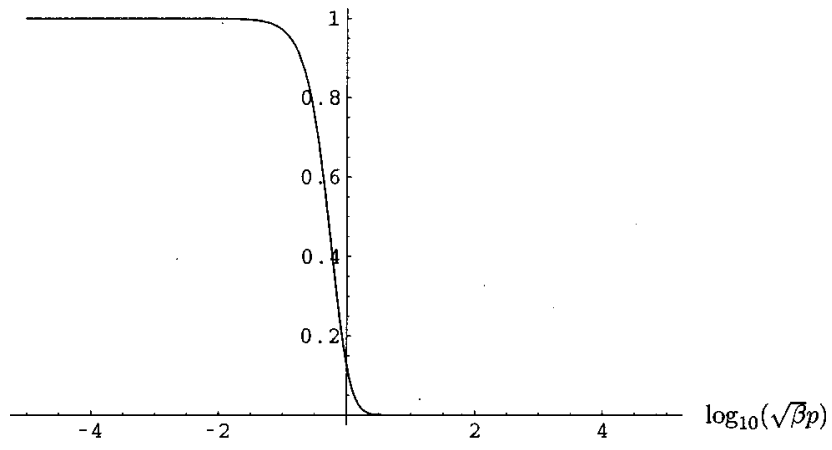

FIG. 1. The behavior of the weight factor $\left[1+(\sqrt{\beta} p)^{2}\right]^{-3}$.

then, the sum over all momentum states per unit volume is (up to some prefactors)

$$
\begin{aligned}
\Lambda(m) & =\int \frac{d^{3} \mathbf{p}}{\left(1+\beta p^{2}\right)^{3}}\left[\frac{1}{2} \sqrt{p^{2}+m^{2}}\right] \\
& =2 \pi \int_{0}^{\infty} \frac{p^{2} d p}{\left(1+\beta p^{2}\right)^{3}} \sqrt{p^{2}+m^{2}} \\
& =\frac{\pi}{2 \beta^{2}} f\left(\beta m^{2}\right),
\end{aligned}
$$

where

$f(x)$

$$
= \begin{cases}1+\frac{x}{2(1-x)}+\frac{x^{2}}{4(1-x)^{3 / 2}} \ln \left(\frac{1-\sqrt{1-x}}{1+\sqrt{1-x}}\right) & (x \leqslant 1), \\ 1-\frac{x}{2(x-1)}+\frac{x^{2}}{2(x-1)^{3 / 2}} \tan ^{-1} \sqrt{x-1} & (x \geqslant 1) .\end{cases}
$$

$f(x)$ is a monotonically increasing function which behaves asymptotically as

$$
f(x) \sim \sqrt{x}
$$

as can be gleaned from Eq. (31). In the region $0 \leqslant x \leqslant 1$, it is well approximated by

$$
f(x) \approx(1+x)^{0.42} .
$$

In the massless case we obtain

$$
\Lambda(0)=\frac{\pi}{2 \beta^{2}} .
$$

As expected, due to the strong suppression of the density of states at high momenta, the cosmological constant is rendered finite with $1 / \sqrt{\beta}$ acting effectively as the UV cutoff. This result is in strong contrast to conventional calculations where the UV cutoff is an arbitrary scale which must be 

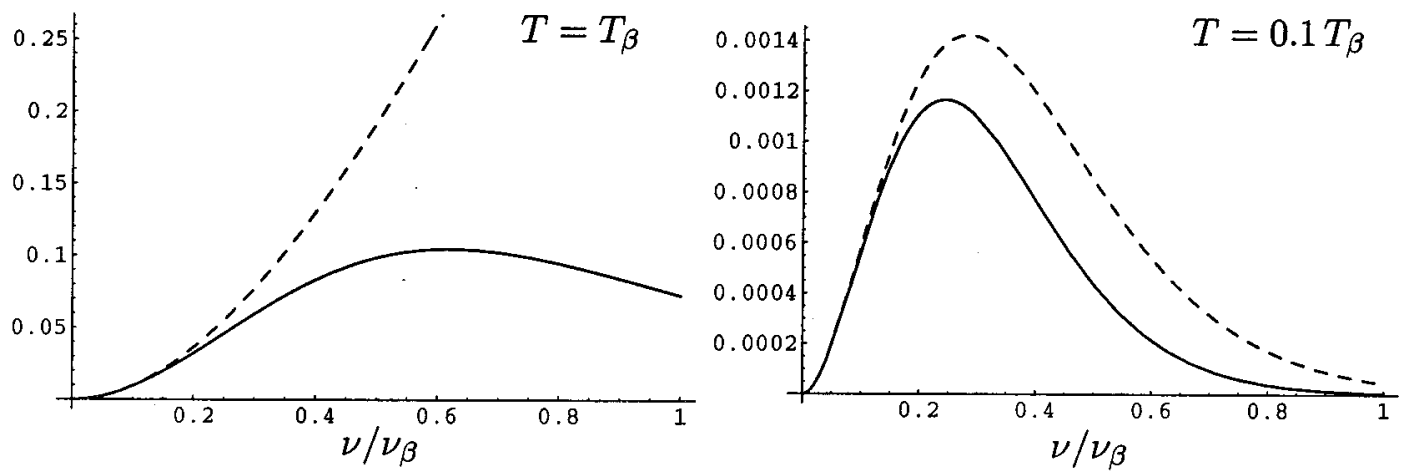

FIG. 2. The shape of the blackbody radiation spectrum with (solid line) and without (dashed line) the damping factor $\left[1+\left(\nu / \nu_{\beta}\right)^{2}\right]^{-3}$ at temperatures $T=T_{\beta}$ (left) and $T=0.1 T_{\beta}$ (right).

introduced by hand, and where one must assume that the physics beyond the cutoff does not contribute.

Unfortunately, since $1 / \sqrt{\beta}$ is the string mass scale, which we expect to be of the order of the Planck mass $M_{p}$, this does not solve the cosmological constant problem. This is true even if the Planck mass $M_{p}$ were as low as a TeV as suggested in models with large extra dimensions [16].

\section{B. The blackbody radiation spectrum}

Taking into account the weight factor Eq. (29), the average energy in the EM field per unit volume at temperature $T$ is

$$
\begin{aligned}
\bar{E} & =2 \int \frac{d^{3} \mathbf{k}}{(2 \pi)^{3}\left[1+\beta(\hbar k)^{2}\right]^{3}} \frac{\hbar k c}{e^{\hbar k c / k_{B} T}-1} \\
& =\frac{8 \pi}{c^{3}} \int_{0}^{\infty} d \nu \frac{1}{\left[1+\beta(h \nu / c)^{2}\right]^{3}}\left(\frac{h \nu^{3}}{e^{h \nu / k_{B} T}-1}\right) \\
& \equiv \int_{0}^{\infty} d \nu u_{\beta}(\nu, T) .
\end{aligned}
$$

We see that the blackbody radiation spectrum is damped at high frequencies close to the cutoff scale:

$$
u_{\beta}(\nu, T)=\frac{1}{\left[1+\left(\nu / \nu_{\beta}\right)^{2}\right]^{3}} u_{0}(\nu, T), \quad \nu_{\beta} \equiv \frac{c}{h \sqrt{\beta}}
$$

Here

$$
u_{0}(\nu, T)=\frac{8 \pi h \nu^{3}}{c^{3}} \frac{1}{e^{h \nu / k_{B} T}-1},
$$

is the regular spectral function.

To see the effect of this damping on the shape of the spectral function, we plot the functions

$$
\begin{aligned}
& f_{0}(\nu, T) \equiv \frac{\left(\nu / \nu_{\beta}\right)^{3}}{e^{\left(\nu / \nu_{\beta}\right)\left(T_{\beta} / T\right)}-1}, \\
& f_{\beta}(\nu, T) \equiv \frac{1}{\left[1+\left(\nu / \nu_{\beta}\right)^{2}\right]^{3}} f_{0}(\nu, T),
\end{aligned}
$$

for several values of the temperature $T$ in Figs. 2 and 3. The temperature $T_{\beta}$ in the definition of $f_{0}(\nu, T)$ is defined as

$$
T_{\beta} \equiv \frac{c}{k_{B} \sqrt{\beta}} .
$$
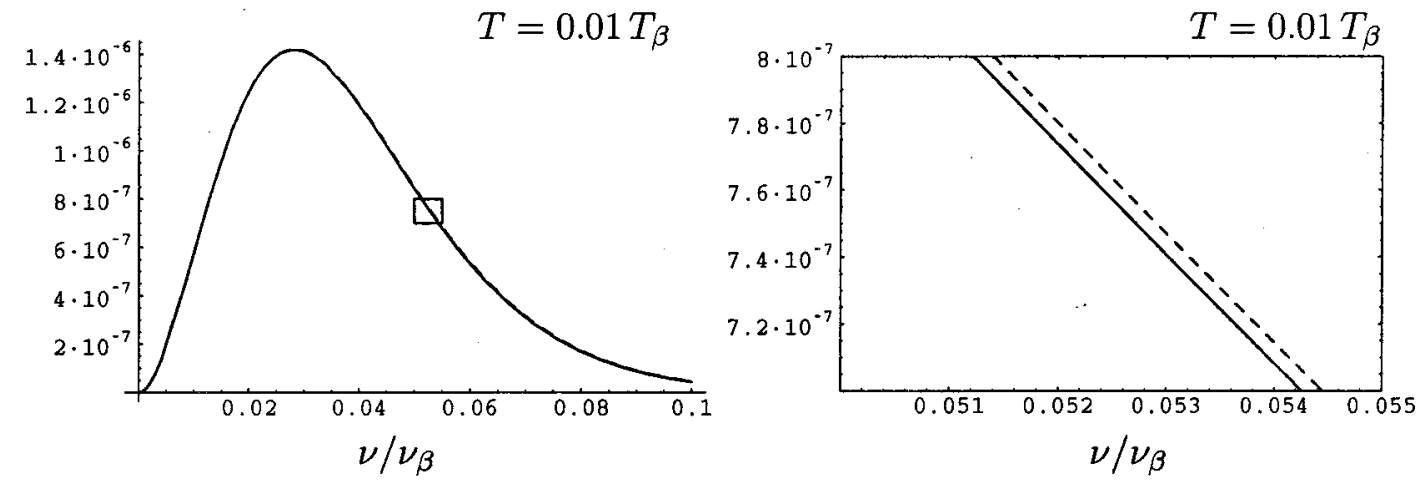

FIG. 3. The shape of the blackbody radiation spectrum with (solid line) and without (dashed line) the damping factor $\left[1+\left(\nu / \nu_{\beta}\right)^{2}\right]^{-3}$ at temperature $T=0.01 T_{\beta}$. The graph on the right is the blowup of the region inside the box on the left. 
As is evident from the figures, the distortion to the blackbody radiation is undetectable unless the temperature is within a few orders of magnitude below $T_{\beta}$. Given that the decoupling temperature is on the order of an $\mathrm{MeV}$ [17], we do not expect the spectrum of the cosmic microwave background (CMB) to be affected in any observable way, even if $1 / \sqrt{\beta}$ were as small as a $\mathrm{TeV}[16]$.

\section{DISCUSSION}

We have shown that Eq. (1) and its higher dimensional extensions imply that the density of states is naturally suppressed in the ultraviolet. This suppression renders the cosmological constant finite, without affecting the blackbody radiation spectrum at observable temperatures. The cosmological constant is still too large, however, since it is proportional to $1 / \beta^{2} \sim M_{p}^{4}$. This scaling is to be expected since $1 / \sqrt{\beta}$ is the only scale present in the problem.

Nevertheless, we believe that our result is a major improvement over previous results and points to a new direction from which we may approach the cosmological constant problem. The reason why conventional calculations were obtaining an infinite result can be identified as a case of overcounting of states. If string theory does indeed lead to Eq. (1) and the resulting suppression of the density of states at high momenta, the number of states in the UV is not as numerous as conventional calculations assume.

The smallness of the cosmological constant can then be interpreted as a sign that we are still overcounting the number of states in our calculation and the density of states should be further suppressed. Whatever the method of suppression, this would necessarily entail the introduction of an additional characteristic scale, $M_{c}$, other than $1 / \sqrt{\beta}=M_{p}$, in which case the dependence of the cosmological constant on the scales can be expressed as $\Lambda \sim M_{p}^{4} F\left(M_{c} / M_{p}\right)$ with $F(1)=1$.

One method we could use would be to modify the righthand side of Eq. (1) to further suppress the higher momen- tum states [20]. However, that would not work since all that would do would be to change the "effective" value of the UV cutoff from $M_{p}$ to $M_{c}$. $\left[F \sim\left(M_{c} / M_{p}\right)^{4}\right]$. So the additional suppression must occur in a more subtle fashion.

Banks [11] has argued that $F \sim\left(M_{c} / M_{p}\right)^{8}$ with $M_{c}$ $\sim 1 \mathrm{TeV}$ and $M_{p} \sim 10^{19} \mathrm{GeV}$ would reproduce the correct value of the cosmological constant. But the exact mechanism which would lead to such a form for $F$ remains elusive. (See also Ref. [18] for a related discussion.)

At this point, we note that if string theory is the correct theory of gravity, modifying the commutation relations alone would not properly take into account all of its potential effects. It is possible that the holographic principle [19] could be of help here. Consideration of holography in a cosmological background might naturally provide another scale other than $1 / \sqrt{\beta}$, namely the size of the cosmological horizon, the Hubble radius $H$, related to the cosmological constant as $H^{2} \sim 1 / \Lambda$. It is conceivable that due to the correct implementation of the holographic principle in a cosmological situation, the number of fundamental degrees of freedom contributing to the vacuum energy is determined by the density of states above some very large momentum [which by the UV/IR correspondence (2) would be related to the degrees of freedom at distances of the order of the cosmological horizon]. If indeed the density of states is strongly suppressed at high momenta, as argued in this paper, then the effective number of degrees of freedom contributing to the vacuum energy density would be very small. While these considerations are highly speculative, they seem to point to a new promising way to approach the cosmological constant problem.

\section{ACKNOWLEDGMENTS}

We would like to thank Vijay Balasubramanian, Per Berglund, Will Loinaz, Asad Naqvi, Koenraad Schalm, Gary Shiu, Joseph Slawny, and Matthew Strassler for helpful discussions. This research is supported in part by a grant from the US Department of Energy, DE-FG05-92ER40709.
[1] L. N. Chang, D. Minic, N. Okamura, and T. Takeuchi, Phys. Rev. D 65, 125027 (2002).

[2] D.J. Gross and P.F. Mende, Nucl. Phys. B303, 407 (1988); Phys. Lett. B 197, 129 (1987); D. Amati, M. Ciafaloni, and G. Veneziano, ibid. 216, 41 (1989); Int. J. Mod. Phys. A 3, 1615 (1988); Phys. Lett. B 197, 81 (1987); E. Witten, Phys. Today 49, 24 (1997); see also G. 't Hooft, Int. J. Mod. Phys. A 11, 4623 (1996); S. de Haro, J. High Energy Phys. 10, 023 (1998).

[3] A. Kempf, Phys. Rev. D 63, 083514 (2001); A. Kempf and J.C. Niemeyer, ibid. 64, 103501 (2001).

[4] J. Polchinski, Phys. Rev. Lett. 75, 4724 (1995); M.R. Douglas, D. Kabat, P. Pouliot, and S.H. Shenker, Nucl. Phys. B485, 85 (1997).

[5] T. Yoneya, Int. J. Mod. Phys. A 16, 945 (2001); T. Yoneya, hep-th/9707002; M. Li and T. Yoneya, Phys. Rev. Lett. 78, 1219 (1997); M. Li and T. Yoneya, hep-th/9806240; H. Awata, M. Li, D. Minic, and T. Yoneya, J. High Energy Phys. 02, 013
(2001); D. Minic, Phys. Lett. B 442, 102 (1998).

[6] T. Yoneya, Prog. Theor. Phys. 103, 1081 (2000).

[7] L. Susskind and E. Witten, hep-th/9805114; A.W. Peet and J. Polchinski, Phys. Rev. D 59, 065011 (1999).

[8] M.R. Douglas and N.A. Nekrasov, Rev. Mod. Phys. 73, 977 (2002).

[9] C.M. Hull, J. High Energy Phys. 07, 021 (1998); 11, 017 (1998); V. Balasubramanian, P. Horava, and D. Minic, ibid. 05, 043 (2001); E. Witten, hep-th/0106109; A. Strominger, J. High Energy Phys. 10, 034 (2001).

[10] A. Strominger, J. High Energy Phys. 11, 049 (2001); V. Balasubramanian, J. de Boer, and D. Minic, Phys. Rev. D 65, 123508 (2002).

[11] T. Banks, Int. J. Mod. Phys. A 16, 910 (2001); For other closely related attempts to understand the cosmological constant problem consult, for example, T. Banks, hep-th/9601151; A.G. Cohen, D.B. Kaplan, and A.E. Nelson, Phys. Rev. Lett. 
82, 4971 (1999); P. Horava and D. Minic, ibid. 85, 1610 (2000); N. Arkani-Hamed, S. Dimopoulos, N. Kaloper, and R. Sundrum, Phys. Lett. B 480, 193 (2000); S. Kachru, M. Schulz, and E. Silverstein, Phys. Rev. D 62, 045021 (2000).

[12] Both observational constraints on and theoretical approaches to the cosmological constant are reviewed in S.M. Carroll, Living Rev. Relativ. 4, 1 (2001); for some theoretical perspectives, see S. Weinberg, astro-ph/0005265; Rev. Mod. Phys. 61, 1 (1989); E. Witten, hep-ph/0002297.

[13] R. Easther, B.R. Greene, W.H. Kinney, and G. Shiu, Phys. Rev. D 64, 103502 (2001); hep-th/0110226; see also L. Mersini, M. Bastero-Gil, and P. Kanti, Phys. Rev. D 64, 043508 (2001); M. Bastero-Gil and L. Mersini, ibid. 65, 023502 (2002); M. Bastero-Gil, P. H. Frampton, and L. Mersini, ibid. 65, 106002 (2002).
[14] A similar observation was made in M. Lubo, hep-th/0009162. [15] A. Kempf, G. Mangano, and R.B. Mann, Phys. Rev. D 52, 1108 (1995); A. Kempf, J. Phys. A 30, 2093 (1997).

[16] N. Arkani-Hamed, S. Dimopoulos, and G. Dvali, Phys. Lett. B 429, 263 (1998); Phys. Rev. D 59, 086004 (1999); I. Antoniadis, N. Arkani-Hamed, S. Dimopoulos, and G. Dvali, Phys. Lett. B 436, 257 (1998).

[17] See, for instance, G.F. Smoot and D. Scott, Eur. Phys. J. C 15, 145 (2000).

[18] P. Berglund, T. Hubsch, and D. Minic, hep-th/0112079; also, hep-th/0201187.

[19] G. 't Hooft, gr-qc/9310026; L. Susskind, J. Math. Phys. 36, 6377 (1995).

[20] Depending on the modification, the cosmological constant may be rendered divergent. For instance, if we adopt the commutation relations used by Kempf in Ref. [3], the cosmological constant will diverge logarithmically. 